\title{
"I'm Not a Chatbot": An Empirical Investigation of Humanized Profiles of Social Media Customer Service Representatives
}

\author{
Huai-Tzu Cheng \\ Louisiana State University \\ hchen53@1su.edu
}

\author{
Yang Pan \\ Tulane University \\ ypan8@tulane.edu
}

\begin{abstract}
While artificial intelligence is robotizing customer service at an unprecedented pace, there is great concern that robotized customer service could undermine customer satisfaction. This study searches for a solution that humanizes customer service to address this concern. Aiming to increase humanization, U.S. telecom giant T-Mobile recently added personal identities to its customer service representatives' profiles on Twitter. Here, we examine the effect of humanized profiles on customers' expressions of emotion or complaints via public tweets. The study provides novel insight explaining why customers are more likely to express positive emotions and fewer complaints if they are interacting with customer service representatives with a humanized profile on a social media platform. Interestingly, this effect is stronger among female users. We also discuss the implications for research and practice.
\end{abstract}

\section{Introduction}

Artificial intelligence (AI) is increasingly robotizing customer service at an unprecedented pace. From automated messages to AI-based chatbots, robotization empowers companies to better support their customers' needs at more touchpoints along their journey. Advanced systems powered by AI, such as chatbots, make around-the-clock interactions widely available. AI-based chatbots bring numerous advantages to the customer service industry: 24/7 availability, handling tedious and repetitive tasks, supplying quick answers to simple questions, providing instant responses, reducing labor costs for human customer service representatives, and so on. Recent industrial reports have shown that, by 2020, more than $85 \%$ of all customer support communications would be conducted without engaging any customer service representatives, and 55\% of established companies have either begun making investments in the potential of AI or are planning to do so by 2020 [1]. Robotization is the inevitable future of customer service.

Despite the above, one great concern associated with robotized customer service is that it could undermine customer satisfaction. What do customers actually think about chatbots? AI-based chatbots do not always lead to a pleasant experience when customers are seeking support. According to a Forrester Research report, $54 \%$ of U.S. online consumers believe that interacting with a chatbot has a negative impact on their quality of life [2]. A recent study by $\mathrm{CGS}^{1}$ examined consumers' preferences for service engagements, and specifically addressed chatbot use. They found that, despite today's technology-dependent environment, consumers still prefer human agents over chatbots for their customer service engagements [3]. Customers feel frustrated when a bot does not always understand them, and they complain that companies that use bots seem to shrug off the problems associated with them [4]. The concern that robotization is driving customers away has led some companies to reconsider whether they are moving too fast to force chatbots on their customers at the cost of their customers' satisfaction.

Customer satisfaction is particularly important on social media platforms, which have become increasingly popular means for brands to deliver customer service and for customers to interact with brands. According to a New York Times article, customers have gradually found that using social media platforms like Twitter and Facebook is a more effective way of getting attention from airlines, for example, compared to contacting their customer service departments via telephone or email [5]. An estimated $67 \%$ of consumers now use social media platforms when seeking a resolution to their customer service issues [6]. At the same time, social media makes it easier than ever for customers to share information, and for it to be shared among all customers. Brands operating on

\footnotetext{
1 Computer Generated Solutions is a business applications, learning,
} and outsourcing services company. 
social media must not only address their customer who is interacting with them on social media, but also anyone who might be a witness to the public exchange. A high satisfaction rate among one's customers promotes the brand's image and fosters brand loyalty. However, negative feelings among customers can erode the brand's reputation in a disruptive way.

As social media becomes the main point of customer contact, and in recognition that maintaining positive levels of customer satisfaction on social media is crucial for any brand, we must ask how we can cultivate positive experiences on social media platforms concerning customer service interactions. Here, we seek the answer by humanizing customer service, especially in this time of fast-paced robotization. Today's social media platforms do not make it easy for customers to determine whether or not they are chatting with a chatbot or a real person; humanized elements increase customers' beliefs that they are chatting with and being taken care of by a real human, therefore they increase customers' happiness levels. As John Legere, the former Chief Executive Officer of T-Mobile US, states, "TForce (T-Mobile's social media specialists) is a team of real people. Not Bots! ... when customers know they're speaking with a real person-and not some humancyborg relations - they're happier!" [7]. This motivates our study. Formally, we seek to answer the research question: Does the use of humanized customer service on social media channels increase customers' positive emotions and reduce complaints on social media?

To answer this question, we conducted a field study by collecting data from the internet's largest social media platform-Twitter. On February 22, 2017, TMobile's official customer service Twitter account adopted a new feature aiming to increase humanization by adding personal identity information to its customer service representatives' profiles, so customers know they are talking to a live person, not a chatbot. We use this change as an exogenous event for all Twitter users and examine the impact of humanized profiles on emotional expressions or complaints via public tweets. We adopted a difference-in-differences (DID) specification and obtained tweets mentioning the official customer service Twitter accounts of four major U.S. telecom companies, AT\&T Cares, Verizon Support, Sprint Care, and T-Mobile Help, for three months before and after this event. Our empirical findings show that humanized customer service leads to an increase in positive sentiments expressed in public tweets and fewer complaint tweets. Interestingly, this effect is stronger for female users than for males.

\section{Literature Review}

\subsection{Customer Service on Social Media}

There are two main streams in the literature related to customer service on social media. The first concerns communication channel preferences, firms' service differentiation, and their response strategies. Customers are able to choose to use public conversations (public posts/tweets) or private conversations (private direct messages) to communicate with firms on social media platforms. He et al. [8] investigated communication channel preferences for service interactions on Twitter from both the customer and airline perspectives. Prior works have suggested that customer service agents working on social media treat customers differently than those interacting via other channels. For instance, Gunarathne et al. [9] suggest that the phenomenon of a customer service differential exists on Twitter by demonstrating that customers are prioritized according to their social status; they also showed that a bystander effect exists within the social media customer service context. In a different study, Gunarathne et al. [10] reported on the impact of racial identity on social media customer service on Twitter. Another relevant work, by Hu et al. [11], showed that the politeness of tweets also affects firms' response strategies on Twitter.

The second stream concerns customer satisfaction with social media-based customer service. Huang et al. [12] suggest that customers who receive a brand's response to their tweets are more likely to pay more, to choose the brand more often in the future, and/or will recommend the brand to others. Other studies have determined that customers with high social status (those seen as more influential) on social networks are more likely to be satisfied with the solution provided by social media-based customer service [13]. Meanwhile, polite customers are more likely to be satisfied with firms' responses by expressing positive sentiments in their following tweets [11].

It should be noted that, although the firms' customer service efforts on social media sites improve the relationships between firms and customers, sometimes prior complaint experiences encourage more complaints later [14]. Another study reported that a customer's previous complaint experiences with a firm lead to lower satisfaction with the final complaint result, compared to customers without a previous complaint experience [13].

\subsection{Social Presence}

Social presence theory has been the focus of many studies in the field of communications. Tu and McIsaac [15] defined social presence in computer mediated communication (CMC) environments as the degree of 
feeling, perception, and reaction to another. Later, Biocca et al. [16] extended the definition of social presence to include "the sense of being with another" or "the sense of being together" in a virtual environment. The characteristics of CMC environments are different than those of face-to-face environments, because people are not typically able to see their actual communication partner. Hence, social presence can be regarded as a critical factor that affects interactions on CMC.

For the purposes of our research, we set the limits of our exploration of social presence in CMC to include only the aspects of social cues in CMC. Prior studies have stated that the absence of identity cues (social context cues) that contain the demographic or personal characteristics of communicators is a major characteristic of CMC, compared with traditional faceto-face communication [17, 18]. Sproull and Kiesler [18] observed that, within text-based CMC, the lack of social context cues impairs the quality of interpersonal communication and leads to dehumanized perceptions of the communicating partner [19].

The visual representation of a communication partner is one of the factors that affects social presence in virtual environments. Prior research has suggested that, when a visual representation is present in CMC interactions, people are more likely to perceive a higher degree of social presence from the communication partner [20]. Tanis and Postmes [21] studied the effect of social cues on impression formation and observed that, when discussion partners convey social cues by displaying their pictures or a short biography, people are more likely to form positive impressions of them and have reduced feelings of ambiguity related to their personal impressions of their discussion partners. Another relevant work, by Feng et al. [17], examined the influence of user profiles containing identity cues on the quality of received responses in the setting of a virtual online forum. They found that people perceived a higher degree of social presence when a user's profile contained cues about their personal identity, such as a photo and first name. In the context of social media, several studies have used personal profiles to evaluate the effect of social cues on social media users. They have suggested that social cues positively affect people's attitudes and perceptions toward users on social media platforms $[22,23]$.

Most of the studies examining how social cues affect peoples' perceptions of others in CMC environments were conducted in a laboratory setting. Our study aims to extend this line of inquiry by using a natural experiment approach and examining the impact of social cues in service representatives' profiles on customers' emotional expressions and complaints in the social media setting. To the best of our knowledge, no previous study has investigated this phenomenon in the context of customer service delivered on a social media platform. Hence, our paper offers unique contributions to this stream of literature.

\section{Research Method}

\subsection{Background}

On February 22, 2017, Twitter launched a new customer service feature for Twitter Business accounts, allowing users to create a custom Twitter profile (referred to hereinafter as a "profile") featuring personal identity information when interacting with customers. This service aimed to provide a more humanized customer service experience, so customers would know they were talking to a person and not a chatbot. TMobile, one of the largest mobile communications companies in the United States, was among the first few companies, and the only telecom carrier in the U.S., to adopt the new feature for its official customer service account, T-Mobile Help. It did so the day the feature became available.

Before the new humanized profile features were adopted, customers could only see the T-Mobile logo and a customer service representative's name or initials when viewing a T-Mobile Help's customer service tweet (customer service representatives' responses to public tweets), as shown in Figure 1(a). In online communication, and especially in a time of increasing robotization, using the company logo and the customer service representative's name in customer service tweets may still lead customers to wonder whether or not they are communicating with a human or a chatbot.

After adopting the new profile feature, T-Mobile Help's customer service tweets displayed a profile with personal identity information, including an agent's profile picture, full name, and a short biography (as shown in Figure 1(b)). Customers could see the profile of the customer service representative they were communicating with. This helped them to know they were talking with a real person [24].

Once a conversation moves to direct messages (DM), customers can also see the service representative's profile photo and full name. Figure 2 (a) shows what customers could see when chatting with $\mathrm{T}$ Mobile Help in DM before this new feature was adopted. By contrast, Figure 2 (b) illustrates shows the view after the new profile features were adopted.

T-Mobile Help's adoption of the humanized profile provides us an opportunity to examine the causal effect of humanization in customer service on customers' expressions of emotion on social media platforms. Since T-Mobile Help's adoption of this new feature is an exogenous event for Twitter users, we can exploit it as a natural experiment setting to conduct our study. Specifically, we adopt a DID estimation to assess the 
impact of humanized profiles of service representatives on customers' sentiments and complaints in public Twitter posts.

\subsection{Data}

To empirically estimate the effect of T-Mobile Help's humanized profile on customers' emotional expressions in public tweets, we used the Twitter API to collect tweets mentioning the official customer service accounts of four major U.S. telecom companies, AT\&T Cares, Verizon Support, Sprint Care, and T-Mobile Help, from November 2016 through May 2017. We retained all tweets posted three months before and after the adoption date of T-Mobile's new customer service profiles, February 22, 2017. Figure 3 illustrates the timeline of our natural experiment setting. The actions by T-Mobile Help constitute the treatment group, while the actions by AT\&T Cares, Verizon Support, and Sprint Care during the same period serve as the control group. We selected AT\&T, Sprint, and Verizon as our control group because they, along with T-Mobile, were the major wireless carriers/operators in the United States between 2011 and 2019 [25].

It should be noted that the official Twitter customer service accounts of these four telecom companies are all operated by human customer service Team. ${ }^{2}$ The difference between the treatment and control groups was the use of humanized profiles for customer service representatives. In the control group, the customer service representatives did not have humanized profiles; instead, they used their brand logo as their profile picture and signed their customer service tweets using their name or initials (this is similar to Figure 1(a)). By contrast, the customer service representatives in the treatment group used a humanized profile that contained their personalized profile picture, full name, and a short biography, as shown in Figure 1(b).

Additionally, the tweets we collected were consolidated into distinct categories of dialogues. We excluded telecom carrier-initiated dialogues, which were more likely to be advertisements, and focused instead on customer-initiated dialogues, potentially capturing emotional responses from customers. Overall, the data set contained 105,286 user-initiated dialogues. We also collected timestamps; tweet content; and Twitter users' profile information, such as the person's number of followers and number of accounts followed. Finally, we created several indicator variables based on the tweets' metadata.

\footnotetext{
2 We searched for the press release for articles related to Twitter customer service account of AT\&T, Verizon, Sprint and T-Mobile.
}

\subsection{Measures}

The study's main outcome variables are measures of emotional expressions and complaints. To ascertain the emotional expressions in tweets, we first measured the linguistic features of each tweet using a text analysis application called Linguistic Inquiry and Word Count (LIWC), which was developed in 2015. LIWC is a commonly used computer-based text analysis tool for exploring the psychological meaning of words. It can accurately identify emotions in language use [26]. It has also been applied to many works in the information systems literature related to sentiment analysis [27, 28]. Our primary linguistic feature in this study is the LIWC's analysis of emotional tone. Emotional tone was measured by a sentiment score. A high sentiment number reflects a more positive attitude in the text, while a low number shows more anxiety, sadness, or the expression of an unfriendly attitude [29].

Next, we constructed a complaint measure based on a machine learning technique using tweets we collected from Twitter. We classified user-initiated tweets separately from all other types of tweets. To determine efficiently whether a tweet was a complaint or not, we adopted a supervised machine learning algorithm to build a complaint classifier. Specifically, our procedure was as follows. First, 1500 tweets were randomly selected from the data set. The authors worked independently to evaluate these tweets as a complaint or non-complaint. If a tweet referred to service or a product inquiry, contained a compliment, and there were no negative terms used in it, it was categorized as a noncomplaint tweet. If a tweet expressed dissatisfaction toward a product or service, it was categorized as a complaint tweet. When there was a disagreement, we sought a third person's opinion and used the majority vote to break the tie. Then, we used a support vector machine algorithm to train a classifier with linguistic features (e.g., count vectorizer and n-grams) and applied a 10-fold cross-validation to measure its performance. The precision of our complaint classifier was roughly $85 \%$, which is a satisfactory result. Finally, we applied the classifier to our data set to identify whether each tweet was a complaint or not. The results showed that the percentages of complaint and non-complaint tweets were $57 \%$ and $43 \%$, respectively.

Further, we defined a dummy variable, Post, coded as " 1 " if the date was on or after the humanized profile feature was adopted and coded as " 0 " if the date was before the adoption. Treat was a dummy variable coded as " 1 " if the telecom carrier was T-Mobile Help and coded as "0" if the carrier was AT\&T Cares, Verizon

We did not find any articles mentioned these four telecom companies adopted AI-based chatbot on Twitter. 
Support, or Sprint Care. Our main interest is the interaction terms of Post and Treat, since they capture the effect of the treatment on customers' sentiments and complaints on social media platform. Tables 1 and 2 present the key variables' definitions and summary statistics for the main variables in our data set, respectively.

\section{Empirical Results}

\subsection{Main Results}

We chose DID as our main empirical strategy, because this approach is typically used to estimate the causal effect of a specific treatment intervention and is a popular research design for estimating causal relationships in empirical economics and social science research $[30,31]$. We aimed to examine the impact of a humanized profile that includes a personal identity on customers' emotional expressions and complaints on Twitter. Our data include tweets from three months before and after T-Mobile Help adopted the humanized profile. This specification incorporates telecom carrier and week-fixed effects, allowing us to control effectively for unobserved heterogeneity at the telecom companies and week levels. More specifically, the equation for the DID estimation is as follows:

$\begin{aligned} y_{i t}= & \left.\beta_{0}+\beta_{1} \text { Post }_{i t}+\beta_{2} \text { (Post }_{i t} * \text { Treat }_{i}\right)+ \\ & \beta_{3} \text { Controls }+\alpha_{i}+\text { Week }_{t}+u_{i t}\end{aligned}$

where $y_{i t}$ are Sentiment St $_{\text {and Complaint }}$. Treat $_{i}$ is a dummy variable equal to 1 if the telecom carrier is $\mathrm{T}$ Mobile Help, and 0 otherwise. Post it $_{\text {is }}$ a dummy variable indicating the post-treatment period. Controls include profile, verified, log number of total tweets, followers, following, likes, days registered, and tweet wordcounts. Variables $\alpha_{i}$ and $W_{e e} k_{t}$ are telecom carrier-specific and week-fixed effects, while $u_{i t}$ is the error term. Standard errors are robust and clustered at the telecom carrier level. Our main interest is the coefficient of the interaction term $\beta_{2}$, which captures the influence of a humanized profile on emotional expressions on a social media platform.

The results of the DID estimation with fixed effects are reported in Table 3, Column 1. There, we see the coefficient of the interaction term Post ${ }^{*}$ Treat is positive and significant, indicating customers are more likely to express positive emotions when they are interacting

\footnotetext{
${ }^{3}$ We used LPM, a linear regression on a binary dependent variable, to better estimate the predicted change in probability of complaint tweets. The major concerns of LPM are that the predicted values can be greater than 1 or less than 0 . In our LPM, 99.4\% of the predicted values fell between 0 and 1 . Further, we used the robust standard error to deal with heteroskedasticity.
}

with service representatives with humanized profiles. In particular, the humanized profile adoption increases the sentiment score by 1.99 .

Next, to estimate the effect of the treatment on customer complaints, we apply a logit regression and a linear probability model (LPM) on the DID setting, since our dependent variable is a binary variable equal to 1 if the tweet is a complaint and 0 otherwise. ${ }^{3}$ The estimation results for logit and LPM are reported in Table 3, Columns 2 and 3, respectively. In both Columns 2 and 3, the coefficients of Post*Treat are negative and statistically significant, thus providing evidence that humanized profiles lead to a reduction in complaint tweets. Economically, the humanized profile adoption decreases the odds of getting a complaint tweet by $6 \%$ (Column 2, Logit Model). The predicted percentage of complaint tweets mentioning T-Mobile Help was reduced by $1.3 \%$ after the adoption of the humanized profiles (Column 3, LPM). Overall, our evidence confirms the finding that humanized profiles used by service representatives in customer service tweets lead to an increase in the sentiments of public tweets and fewer complaint tweets. ${ }^{4}$

\subsection{Robustness Check: Testing the Parallel Trend Assumption}

The key assumption of DID is that the trends should be the same in both the control and treatment groups in the absence of the treatment, which is unobservable and impractical to test directly [32]. Following previous DID research [30, 32, 33], we tested the parallel trend assumption during the pretreatment stage and before the change, to ensure the treatment and control groups were comparable. To formally test whether there is a difference in the pre-treatment period between the treatment and control groups, Meyer [33] suggests examining this validity threat by using data from multiple pretreatment periods. We operationalized the pretreatment trend test by following Kumar and Telang [34] and set up two pretreatment periods for the treatment and control groups. Specifically, we estimated Equation (1) for the treatment and control groups during two pretreatment periods. A significant coefficient estimate for the variable Post*Treat would indicate a differential trend in customers' emotional expressions or complaints for the treatment and control groups. These results are reported in Table 4 . We note that, consistently, the coefficients of the interaction term

\footnotetext{
${ }^{4}$ To further examine various time fixed effects on our model, we first used day of week dummies to control within-week variations. We also used day dummies and month dummies to control the day- and monthlevel seasonality separately. Overall, the results are consistent with our main findings.
} 
Post*Treat are statistically insignificant, which indicates that tweets' sentiments and complaints in the treatment and control groups have similar trends in the pretreatment period.

\subsection{Robustness Check: Matching}

In our study, since the users are self-selected to tweet T-Mobile Help, the samples in the treatment group were not randomly selected; therefore, endogeneity concerns emerge. Following Kumar et al. [35], we used a DID approach combined with propensity score matching (PSM) to address the endogeneity concerns and sample selection bias issues. Our matching approach is applied at the Twitter user level, and we matched each user in the treatment group to the most similar user in the control group, based on the profile and tweeting behavior information about the Twitter users. In our matching process, the treated samples were those users who only tweeted to T-Mobile Help, and the untreated samples were users who only tweeted to AT\&T Cares, Verizon Support, or Sprint Care. There were, in all, 5,797 users in the treatment group, based on our selection criteria.

We applied a logit regression with a set of users' observable variables, such as total tweets, followers, following, and likes, to estimate the predicted propensity score. The matching algorithm we used was 1:1 nearest neighbor (NN) matching without replacement. Then, we used two sample t-test results to evaluate the quality of the matches. After matching, we found that there were no significant differences for all covariate means in the two groups. Then, we reapplied the DID model (1) using the new matched sample after the PSM procedure. These results are presented in Table 5. The coefficients of the interaction term Post*Treat are similar to our baseline model, shown in Table 3. These results indicate that adopting a humanized profile increases the sentiment of tweets by 2.7. Moreover, the odds of getting a complaint tweet decrease by $17 \%$ (Column 2, Logit Model), while the predicted percentage of complaint tweets is reduced $3.7 \%$ (Column 3, LPM), after T-Mobile Help's service representatives adopted humanized profiles. ${ }^{5}$

\section{Heterogeneous Treatment Effects}

Previous studies have suggested that, when social cues are given in CMC, gender differences exist in expectations and perceptions [36]. We hypothesized that the relationship between service representatives' humanized profile adoption and customers' sentiments and complaints would be uneven across the genders. 5 Additionally, we applied PSM with one-to-one matching with
replacement and a one-to-two matching algorithm and coarsened
Specifically, we anticipated that the impact of humanized profiles would have a larger effect on females than on males. Since females tend to be more emotionally expressive than males, they may be more likely to be affected by the use of a humanized profile.

To test this assumption, we checked the heterogeneous treatment effects on males and females. We divided Twitter users in our data set into different gender groups, male users, female users, and others (gender non-identifiable), by using a facial recognition technique (Microsoft Azure). Our data set contained 31,715 male users, 22,896 female users, and 50,675 users with an unidentifiable gender. To better examine the difference between females and males, we excluded from this analysis users with an unidentifiable gender. Next, we estimated the following regression equation to investigate whether the impact of service representatives' humanized profile adoption was moderated by gender.

$$
\begin{aligned}
& y_{i t}= \beta_{0}+\beta_{1} \text { Post }_{i t}+\beta_{2} \text { Female }_{i}+\beta_{3} \text { (Post }_{i t} * \\
&\text { Female } \left._{i}\right)+\beta_{4}\left(\text { Treat }_{i} * \text { Female }_{i}\right)+ \\
& \\
& \beta_{5}\left(\text { Post }_{i t} * \text { Treat }_{i}\right)+\beta_{6} \text { (Post }_{i t} * \text { Treat }_{i} * \\
&\text { Female } \left._{i}\right)+\beta_{7} \text { Controls }+\alpha_{i}+\text { Week }_{t}+u_{i t}(2)
\end{aligned}
$$

where $y_{i t}$ refers to Sentiment St $_{\text {it }}$ and Complaint C. $_{i t}$. Female $_{i}$ is a binary variable equal to 1 if the gender of a Twitter user is female, and 0 otherwise. The variable of interest is the coefficient on the three-way interaction term, $\beta_{6}$, which captures the effect of gender on DID effects between the control and treatment groups. The control variables were the same as in DID model (1).

The heterogeneous effect by gender on the sentiments of tweets is reported in Table 6, Column 1. We found the coefficient of the three-way interaction term Post*Treat*Female was positive and statistically significant. In particular, for female users, the adoption of a personalized profile increased the sentiment of tweets by 1.16 . The estimation results of logit and LPM are reported in Table 6, Columns 2 and 3, respectively. We observed significant and negative coefficients of the three-way interaction term Post*Treat*Female. Specifically, for female users, the predicted percentage of complaint tweets decreased by about $5 \%$ (Column 3 , LPM). Overall, these results are consistent with our expectation that a humanized profile has a significantly greater impact on female users, compared to male users.

\section{Conclusion}

Using a unique dataset of public customer tweets from four major U.S. telecom companies' official

exact matching. We found that all of the matching results are consistent with the PSM estimation reported here. 
customer service accounts on Twitter over a period of six months and leveraging machine learning techniques and a natural experiment setting, we investigated whether the presence of humanized customer service profiles has a positive impact on customers' tweeting behaviors, in terms of the linguistic features in tweets and customer complaint tweets. The study provides the novel insight that customers are more likely to express positive emotions and fewer complaints if they are interacting with a service representative with a humanized profile on social media. Interestingly, this effect is stronger among female users. Customers do not intuitively know whether they are communicating with a bot or a real person on a social media platform, so the humanized profile of service representative undoubtedly increases their belief that they are

\section{References}

[1] Zendesk. Gartner examines customer experience innovation for 2017. 2017 [cited 2020 May 2];

Available from:

https://www.zendesk.com/resources/gartnerexplores-customer-experience-innovation-2017/.

[2] Jacobs, I. Forrester's Latest Infographic: Consumers Don't Believe The Chatbot Hype. 2019 [cited 2020 May 2]; Available from: https://go.forrester.com/blogs/forresters-latestinfographic-consumers-dont-believe-the-chatbothype/.

[3] CGS. Chatbots Deliver Speed, But Consumers Still Want Humans. Are We Moving too Quickly to Automation? . 2018 [cited 2020 May 2]; Available from: https://www.cgsinc.com/en/infographics/chatbots -deliver-speed-but-consumers-want-humans.

[4] Elliott, C. Chatbots Are Killing Customer Service. Here's Why. 2018 [cited 2019 November 17]; Available from:

https://www.forbes.com/sites/christopherelliott/2 018/08/27/chatbots-are-killing-customer-serviceheres-why/\#46c996e413c5.

[5] Wolfe, J. Want Faster Airline Customer Service? Try Tweeting. 2018 [cited 2019 November 17]; Available from: https://www.nytimes.com/2018/11/20/travel/airli ne-customer-service-twitter.html.

[6] Ahmed, M. Social Media Customer Service Statistics and Trends [Infographic]. 2017 [cited 2020 May 2]; Available from: https://www.socialmediatoday.com/socialbusiness/social-media-customer-servicestatistics-and-trends-infographic.

[7] Legere, J. May the T-Force Be with You! 2017 [cited 2019 November 17]; Available from: https://www.t-mobile.com/news/may-the-tforcebe-with-you.

[8] He, S., Lee, S.-Y., and Rui, H. Open Voice or Private Message? The Hidden Tug-of-War on Social Media Customer Service. in In Proceedings of the communicating with and being taken care of by a real human. Our paper contributes to a growing stream of studies in information systems focusing on social media customer service and sheds light on the impact of humanized customer service on customers' emotional expressions and complaints on social media platforms. The paper also helps us to better understand the effect of social cues on social media users. This work has important implications for firms providing consumer support through social media channels. One valuable practical implication of the paper is that managers and practitioners should consider adding more humanized elements when delivering customer service through social media, to nudge the positive sentiments expressed in social media posts.

52nd Hawaii International Conference on System Sciences. 2019.

[9] Gunarathne, P., Rui, H., and Seidmann, A., When Social Media Delivers Customer Service: Differential Customer Treatment in the Airline Industry. MIS Quarterly, 2018. 42(2): p. 489-520.

[10] Gunarathne, P., Rui, H., and Seidmann, A. Racial Bias in Social Media Customer Service: Evidence from Twitter. in In Proceedings of the Thirty Ninth International Conference on Information Systems, San Francisco 2018. 2018.

[11] Hu, Y., Tafti, A., and Gal, D. Read This, Please? The Role of Politeness in Customer Service Engagement on Social Media. in In Proceedings of the 52nd Hawaii International Conference on System Sciences. 2019.

[12] Huang, W., Mitchell, J., Dibner, C., Ruttenberg, A., and Tripp, A. How Customer Service Can Turn Angry Customers into Loyal Ones. 2018 [cited 2020 April 25]; Available from: https://hbr.org/2018/01/how-customer-servicecan-turn-angry-customers-into-loyal-ones.

[13] Gunarathne, P., Rui, H., and Seidmann, A., Whose and What Social Media Complaints Have Happier Resolutions? Evidence from Twitter. Journal of Management Information Systems, 2017. 34(2): p. 314-340.

[14] Ma, L., Sun, B., and Kekre, S., The Squeaky Wheel Gets the Grease-An Empirical Analysis of Customer Voice and Firm Intervention on Twitter. Marketing Science, 2015. 34(5): p. 627-645.

[15] Tu, C.-H. and McIsaac, M., The Relationship of Social Presence and Interaction in Online Classes. The American Journal of Distance Education, 2002. 16(3): p. 131-150.

[16] Biocca, F., Harms, C., and Burgoon, J.K., Toward a More Robust Theory and Measure of Social Presence: Review and Suggested Criteria. Presence: Teleoperators and Virtual Environments, 2003. 12(5): p. 456-480.

[17] Feng, B., Li, S., and Li, N., Is a Profile Worth a Thousand Words? How Online Support-Seeker's Profile Features May Influence the Quality of 
Received Support Messages. Communication Research, 2016. 43(2).

[18] Sproull, L. and Kiesler, S., Connections: New ways of working in the networked organization. 1991, Cambridge, MA: The MIT Press.

[19] MacGeorge, E. and Swol, L., eds. The Oxford Handbook of Advice. 2018, Oxford University Press.

[20] Oh, C.S., Bailenson, J.N., and Welch, G.F., $A$ Systematic Review of Social Presence: Definition, Antecedents, and ImplicationsFrontiers in Robotics and AI, 2018. 5: p. 1-35.

[21] Tanis, M. and Postmes, T., Social Cues and Impression Formation in CMC. Journal of Communication, 2003. 53(4): p. 676-693.

[22] Hong, S., TandocJr, E., Kim, E.A., Kim, B., and Wise, K., The Real You? The Role of Visual Cues and Comment Congruence in Perceptions of Social Attractiveness from Facebook Profiles. Cyberpsychology, Behavior, and Social Networking, 2012. 15(6).

[23] Jahng, M.R. and Littau, J., Interacting Is Believing: Interactivity, Social Cue, and Perceptions of Journalistic Credibility on Twitter. Journalism \& Mass Communication Quarterly, 2016. 93(1): p. 38-58.

[24] Perez, S. Twitter debuts custom profiles for businesses' agents, so people don't think they're talking to bots. 2017 [cited 2019 November 17]; Available from: https://techcrunch.com/2017/02/22/twitterdebuts-custom-profiles-for-businesses-so-peopledont-think-theyre-talking-to-bots/.

[25] Statista. Wireless carrier/operator subscriber share in the U.S. 2011-2019. 2020 [cited 2020 March 26]; Available from: https://www.statista.com/statistics/199359/market -share-of-wireless-carriers-in-the-us-bysubscriptions/.

[26] Tausczik, Y.R. and Pennebaker, J.W., The Psychological Meaning of Words: LIWC and Computerized Text Analysis Methods. Journal of
Language and Social Psychology, 2009. 29(1): p. 24-54.

[27] Kim, K., Park, J., Pan, Y., and Zhang, K. Information Disclosure and Crowdfunding: An Empirical Analysis of the Disclosure of Project Risk. 2017. Academy of Management, 2017.

[28] Huang, N., Hong, Y., and Burtch, G., Social Network Integration and User Content Generation: Evidence from Natural Experiments. MIS Quarterly, 2017. 41(4): p. 1035-1058.

[29] Pennebaker, J.W., Booth, R.J., Boyd, R.L., and Francis, M.E., Linguistic Inquiry and Word Count: LIWC2015. 2015, Pennebaker Conglomerates (www.LIWC.net): Austin, TX.

[30] Bertrand, M., Duflo, E., and Mullainathan, S., How Much Should We Trust Differences-in-Differences Estimates? The Quarterly Journal of Economics, 2004. 119(1): p. 249-275.

[31] Lechner, M., The Estimation of Causal Effects by Difference-in-Difference MethodsEstimation of Spatial Panels. Foundations and Trends ${ }^{\circledR}$ in Econometrics, 2010. 4(3): p. 165-224.

[32] Angrist, J.D. and Pischke, J.-S., Mostly Harmless Econometrics: An Empiricist's Companion. 2008, Princeton, NJ: Princeton University Press.

[33] Meyer, B.D., Natural and Quasi-Experiments in Economics. Journal of Business \& Economic Statistics, 1995. 13(2): p. 155-161.

[34] Kumar, A. and Telang, R., Does the Web Reduce Customer Service Cost? Empirical Evidence from a Call Center. Information Systems Research, 2012. 23(3-part-1): p. 721-737.

[35] Kumar, N., Qiu , L., and Kumar, S., Exit, Voice, and Response on Digital Platforms: An Empirical Investigation of Online Management Response Strategies. Information Systems Research, 2018. 29(4): p. 779-1068.

[36] Matheson, K., Social cues in computer-mediated negotiations: Gender makes a difference. Computers in Human Behavior, 1991. 7(3): p. 137-145.

\section{Appendix}

Table1. Variable's Definitions

\begin{tabular}{|l|l|}
\hline \multicolumn{1}{|c|}{ Variables } & \multicolumn{1}{c|}{ Definition } \\
\hline Sentiment & Sentiment score (positive and negative emotions, social processes, and causation expressed in tweets) \\
\hline Complaint & A dummy variable coded as 1 if the tweet is a complaint; 0 if the tweet is not a complaint \\
\hline Post & A dummy variable coded as 1 if the date is on or after treatment; 0 if the date is before the treatment \\
\hline Treat & $\begin{array}{l}\text { A dummy variable coded as 1 if the telecom carrier is T-Mobile Help; 0 if the telecom carrier is AT\&T } \\
\text { Cares, Verizon Support, or Sprint Care }\end{array}$ \\
\hline Total tweets & Number of tweets the user posted \\
\hline Followers & Number of followers the user had \\
\hline Following & Number of people the user followed \\
\hline Likes & Number of tweets the user liked \\
\hline Days registered & Number of user's registered days with a Twitter account prior to the creation of the tweet \\
\hline Profile & $\begin{array}{l}\text { A dummy variable coded as 1 if the user's bio (profile description) or location is publicity available; 0 } \\
\text { otherwise }\end{array}$ \\
\hline Verified & A dummy variable coded as 1 if it is determined to be an account of public interest; 0 otherwise \\
\hline Tweet word count & The word counts per tweet \\
\hline
\end{tabular}


Table 2. Summary Statistics of Variables

\begin{tabular}{|l|c|c|c|c|c|}
\hline \multicolumn{1}{|c|}{ Variables } & Obs. & Mean & Std. Dev. & Min & Max \\
\hline Sentiment & 105,286 & 41.58109 & 37.12543 & 0 & 99 \\
\hline Total tweets & 105,286 & 15936.69 & 41487.22 & 0 & 1918474 \\
\hline Followers & 105,286 & 7083.162 & 186281.6 & 0 & 6450882 \\
\hline Following & 105,286 & 1057.947 & 8989.94 & 0 & 889327 \\
\hline Likes & 105,286 & 7881.185 & 23345.35 & 0 & 763309 \\
\hline Days registered & 105,286 & 2080.439 & 895.6401 & 1 & 3954 \\
\hline Tweet word count & 105,286 & 17.45637 & 6.459404 & 0 & 35 \\
\hline
\end{tabular}

Table 3. Difference-in-Differences Estimation

\begin{tabular}{|c|c|c|c|}
\hline & (1) & $(2)$ & (3) \\
\hline & Sentiment & Complaint Logit & Complaint LPM \\
\hline \multirow[t]{2}{*}{ Post } & $-2.039 *$ & 0.050 & 0.007 \\
\hline & $(0.684)$ & $(0.060)$ & $(0.012)$ \\
\hline \multirow[t]{2}{*}{ Post $*$ Treat } & $1.992 * * *$ & $-0.063 * * *$ & $-0.013 * * * *$ \\
\hline & $(0.206)$ & $(0.008)$ & $(0.001)$ \\
\hline Controls & Yes & Yes & Yes \\
\hline Telecoms FE & Yes & Yes & Yes \\
\hline Week FE & Yes & Yes & Yes \\
\hline Obs. & 105286 & 105286 & 105286 \\
\hline R-squared/Pseudo $\mathrm{R}^{2 *}$ & 0.003 & $0.131 *$ & 0.144 \\
\hline
\end{tabular}

Table 4. Pre-trend Difference-in-Differences Estimations

\begin{tabular}{|l|c|c|c|}
\hline & $(1)$ & $(2)$ & $(3)$ \\
\hline Post & Sentiment & Complaint Logit & Complaint LPM \\
\hline & 0.676 & -0.113 & -0.019 \\
\hline Post $^{*}$ Treat & $(1.536)$ & $(0.074)$ & $(0.013)$ \\
\hline & $\mathbf{- 0 . 0 1 0}$ & $\mathbf{0 . 0 3 3}$ & $\mathbf{0 . 0 0 4}$ \\
\hline Controls & $\mathbf{( 0 . 8 8 4 )}$ & $\mathbf{( 0 . 0 8 5 )}$ & $\mathbf{( 0 . 0 1 6 )}$ \\
\hline Telecoms FE & Yes & Yes & Yes \\
\hline Week FE & Yes & Yes & Yes \\
\hline Obs. & Yes & Yes & Yes \\
\hline R-squared/Pseudo R & 57379 \\
\hline
\end{tabular}

Table 5. PSM Difference-in-Differences Estimations

\begin{tabular}{|c|c|c|c|}
\hline & $(1)$ & $(2)$ & $(3)$ \\
\hline & Sentiment & Complaint Logit & Complaint LPM \\
\hline \multirow[t]{2}{*}{ Post } & $-3.626 * * *$ & $0.187^{*}$ & 0.033 \\
\hline & $(0.599)$ & $(0.098)$ & $(0.018)$ \\
\hline \multirow[t]{2}{*}{ Post*Treat } & $2.727 * * *$ & $-0.197 * * *$ & $-0.037 *$ \\
\hline & $(0.424)$ & $(0.187)$ & $(\mathbf{0 . 0 3 3 )}$ \\
\hline Controls & Yes & Yes & Yes \\
\hline Telecoms FE & Yes & Yes & Yes \\
\hline Week FE & Yes & Yes & Yes \\
\hline Obs. & 61727 & 61727 & 61727 \\
\hline R-squared/Pseudo R $\mathrm{R}^{2 *}$ & 0.005 & $0.122 *$ & 0.138 \\
\hline
\end{tabular}

Table 6. Heterogeneous Treatment Effects, By Gender

\begin{tabular}{|l|c|c|c|}
\hline & $(1)$ & $(2)$ & $(3)$ \\
\hline & Sentiment & Complaint Logit & Complaint LPM \\
\hline Post & -1.524 & 0.060 & 0.007 \\
\hline & $(2.368)$ & $(0.097)$ & $(0.019)$ \\
\hline Female & -0.693 & $0.281^{* * *}$ & $0.046^{* * *}$ \\
\hline & $(0.602)$ & $(0.050)$ & $(0.006)$ \\
\hline
\end{tabular}




\begin{tabular}{|l|c|c|c|}
\hline Post $*$ Female & $-0.966 * * *$ & $0.146^{* * *}$ & $0.023^{* *}$ \\
\hline & $(0.090)$ & $(0.036)$ & $(0.006)$ \\
\hline Treat $*$ Female & $-1.763^{*}$ & $0.188^{* * *}$ & $0.047 * * *$ \\
\hline & $(0.602)$ & $(0.041)$ & $(0.005)$ \\
\hline Post $*$ Treat & $\mathbf{1 . 3 5 7 * * *}$ & $\mathbf{0 . 0 6 4} * * *$ & $\mathbf{0 . 0 1 2} * * *$ \\
\hline & $\mathbf{( 0 . 0 6 8 )}$ & $\mathbf{( 0 . 0 1 0 )}$ & $\mathbf{( 0 . 0 0 1 )}$ \\
\hline Post $*$ Treat $*$ Female & $\mathbf{1 . 1 5 7} * * *$ & $\mathbf{- 0 . 2 9 3} * *$ & $\mathbf{- 0 . 0 5 2} * * *$ \\
\hline & $(\mathbf{0 . 1 2 7})$ & $(\mathbf{0 . 0 3 3 )}$ & $\mathbf{( 0 . 0 0 5 )}$ \\
\hline Controls & Yes & Yes & Yes \\
\hline Telecoms FE & Yes & Yes & Yes \\
\hline Week FE & Yes & Yes & Yes \\
\hline Obs. & 54611 & 54611 & 54611 \\
\hline R-squared/Pseudo R ${ }^{2 *}$ & 0.004 & $0.137 *$ & 0.150 \\
\hline
\end{tabular}

Robust Standard errors are in parentheses $* * * p<0.01$, ** $p<0.05$, * $p<0.1$

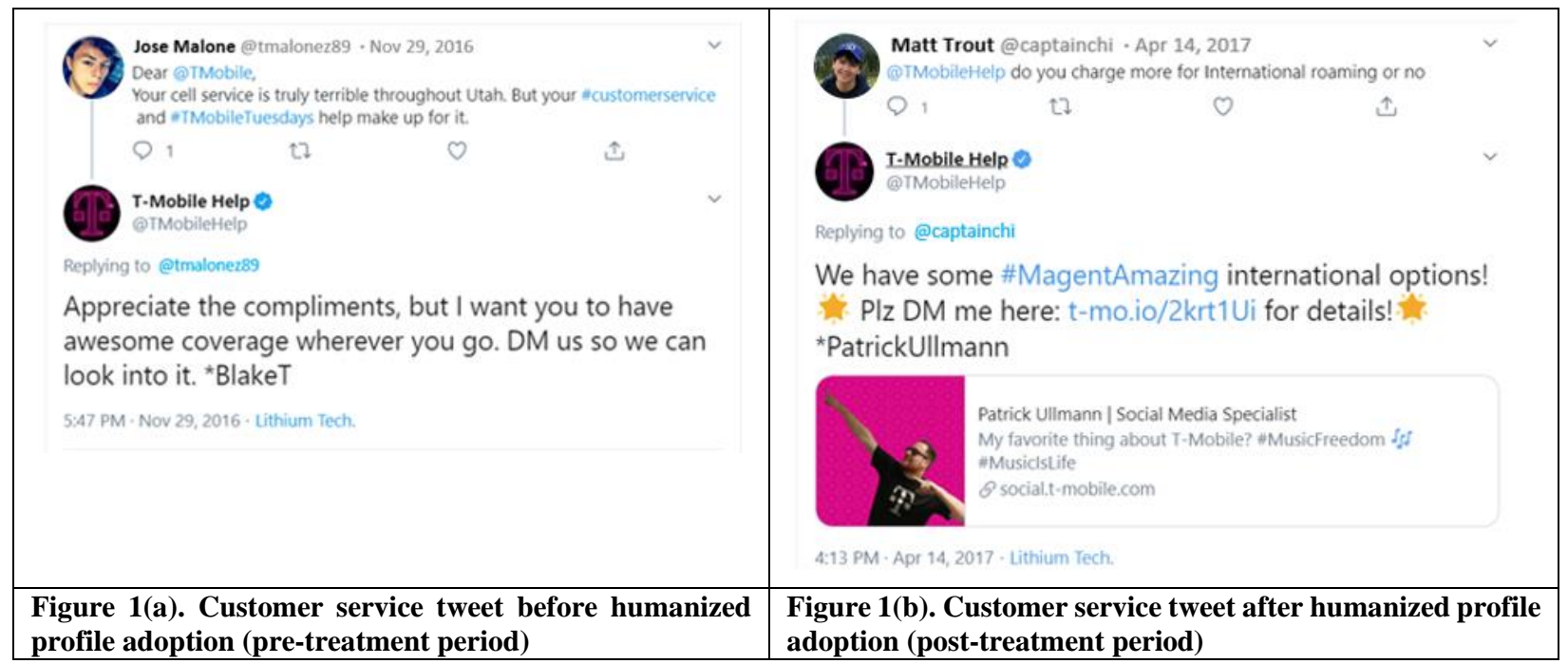

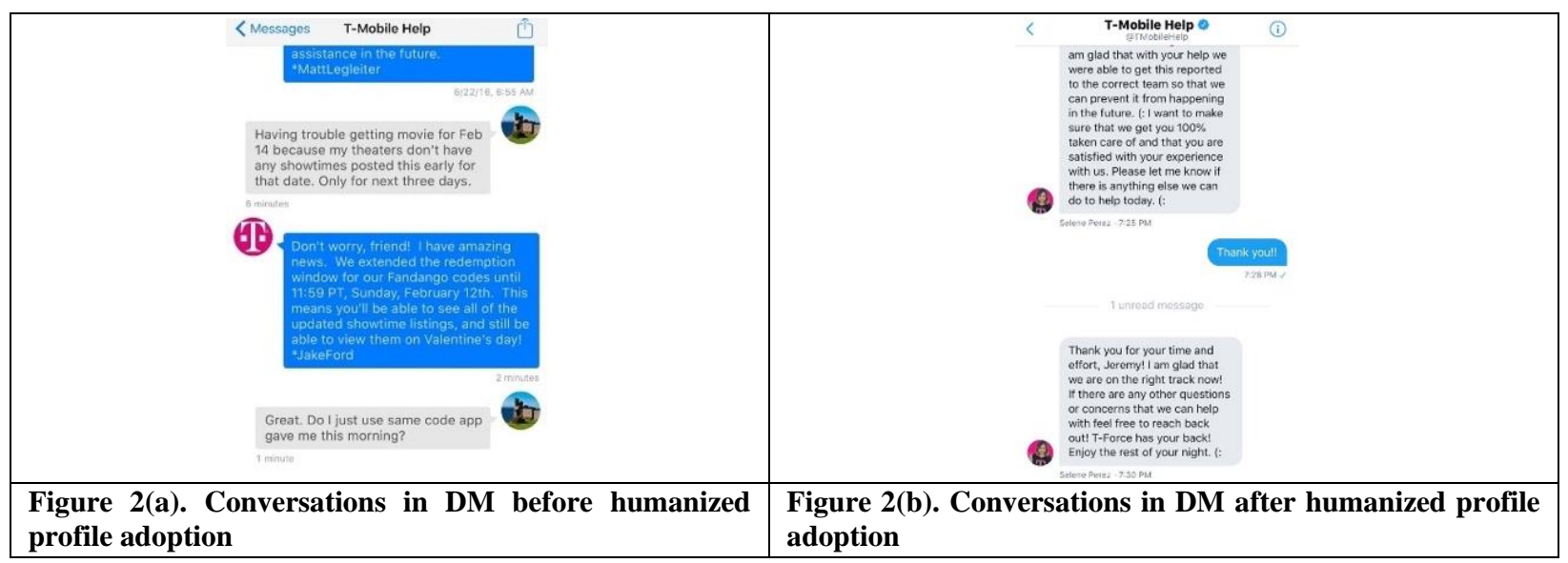

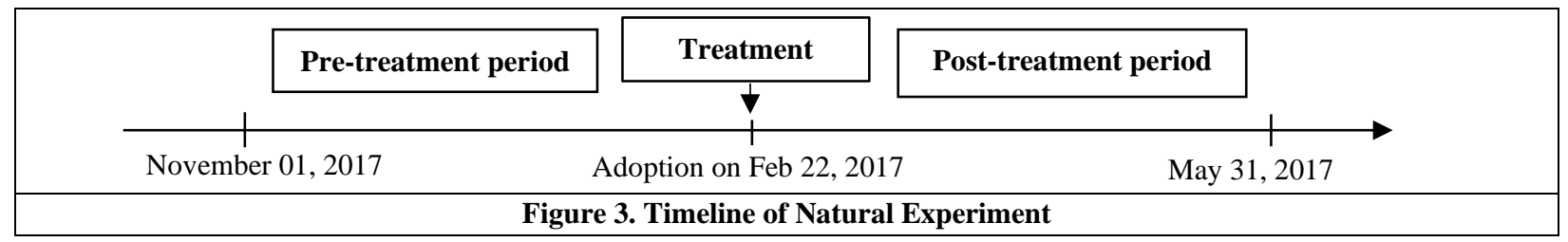

